\title{
Improving the Safety of Pedestrians by Using a Cooperative System
}

\author{
Johannes Morgenroth*, Lars Wolf* \\ S. Macht*, A. Sasse*, P. Hecker*, M. Schack*, M. Vollrath*, U. Seiffert*, K.-O. Proskawetz*, D. Otte ${ }^{\dagger}$ \\ ${ }^{*}$ TU Braunschweig and ${ }^{\dagger}$ Hannover Medical School
}

\begin{abstract}
The number of traffic accidents with injury to persons is still too high. Hence, it is an important question, how the number of accidents with pedestrians can be reduced. We propose to integrate existing technologies and mobile devices into a cooperative system which is able to detect critical situations and execute preventive counteractive measures such as warn traffic participants. The paper starts with an analysis of the different types of accidents in a detailed accident study using the GIDAS database and reports of local accidents with pedestrians. With regard to the different types of accidents it is important to consider the reasons for each situation. The obtained knowledge is used to build general scenarios to determine on effective means to prevent such accidents. Finally, three different system architectures with varying level of capabilities and complexity are designed.
\end{abstract}

Index Terms-pedestrian, protection, localization, communication, vehicle2vehicle, cooperative system

\section{INTRODUCTION}

Significant improvements to lower the number of traffic fatalities have been achieved over the last decades. However the number of accidents with injury to persons is still too high. For example, 336,600 accidents were registered by German police in the year 2005. $42 \%$ of them happened on roads in urban regions and $66 \%$ of the involved persons belonged to the group of non-motorized road users. To improve the traffic safety, daytime running lights are compulsory in the EU for new cars. But this law could lead to a lower visibility of pedestrians and cyclists. Additionally, road vehicles are going to be quieter in the future (moving away from combustion engines to hybrid or purely electrical vehicles) and therefore more difficult to recognize. Hence, it is an important question, how the number of accidents with pedestrians can be reduced under such circumstances.

In a joint project $^{1}$, researchers from different disciplines studied approaches to improve the protection of pedestrians using a cooperative system. The result of this study will be discussed in the following sections.

\section{ACCIDENT STUDY}

As shown in Figure 1, the number of traffic deaths has been lowered significantly since the 90's. Passive safety, protection of the collision partner, road safety education and the adjustment of the list of traffic penalties has decreased the accident severity of accidents between two cars, but despite those improvements the number of traffic deaths of pedestrians and cyclists is still unacceptably high.

\footnotetext{
${ }^{1}$ This work has been supported by EFRE W2-210-2007-0010.
}

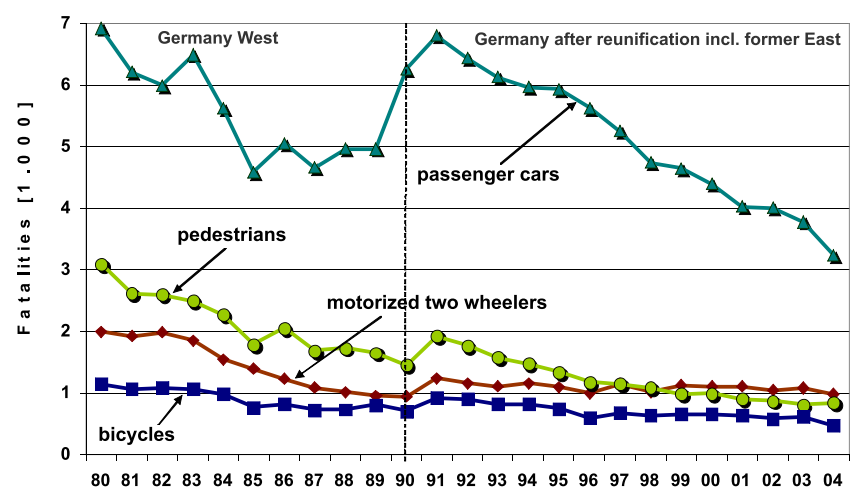

Figure 1. Evolution of the traffic deaths between 1980 - 2004 [1]

To appreciate the capability of a cooperative system for pedestrian safety, a detailed accident study has been performed and will be discussed in the following sections. It will identify critical situations and derive scenarios where technical support can help to reduce the number of accidents or at least mitigate the accident symptoms. This study is based on two data sources, the German In-Depth Accident Study (GIDAS) [2] and some detailed reports of local accidents with pedestrians collected by the police of Braunschweig [3].

\section{A. GIDAS}

Within the GIDAS project, extensive data of accidents has been collected. The employed sampling mechanisms guarantee comparability with federal statistics. The used database consists of 16,337 accidents, in 1,316 of them pedestrians are involved. All accident reports were used to considered different traits of accidents. These traits are:

- primary collision partner

- type of road

- speed limit

- location of the accident

- surface of the road

In $15.1 \%$ of all cases, the cars collide with cyclists and in $7.5 \%$ of all cases with pedestrians. Most accidents happend on roads in urban areas $(71.9 \%)$. This also affects the usual speed limit which was in $78.1 \%$ at $50 \mathrm{~km} / \mathrm{h}$ and in $14.4 \%$ at $30 \mathrm{~km} / \mathrm{h}$. $55.9 \%$ of all accidents happend on straight roads and $23.6 \%$ at crossways. $70.4 \%$ happend on dry roads. This implies that weather does not have a significant impact. 


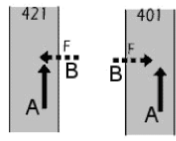

$17,0 \%$

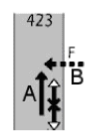

$8,1 \%$

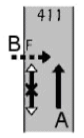

$5,8 \%$

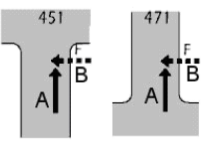

$5,8 \% \quad 3,7 \%$

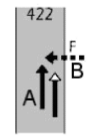

$3,4 \%$

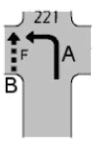

$3,2 \%$
Figure 2. Most frequently occurring types of accidents.

The most frequently occuring types of accidents (see fig. 2) between cars and pedestrians have been identified from the reports. Accident type 421 shows a straight road where the pedestrian steps on the road from the right side, this happened in $17 \%$ of the cases. In the accident type 401 the pedestrian steps from the left side on the road $(8.7 \%)$. A blocked view situation is described in accident type $423(8.1 \%)$ and type $411(5.8 \%)$. Thus, accidents on straight roads seem to be the most important cases to consider.

The speed of collision has been considered in a comparing report and confirmed the assumption, that most of the accidents between passenger cars and pedestrians occur in urban areas. In $90 \%$ of all accidents, the speed of collision was not faster than $60 \mathrm{~km} / \mathrm{h}$. Especially accident type 423, where the driving speed was not higher than $50 \mathrm{~km} / \mathrm{h}$, is representative for an urban scenario.

Finally, the analysis of the reaction time of the driver discovered that merely $50 \%$ (type 421 and 401 ) braked before the collision. In accident type 423, this was significantly higher (76.4\%), but the average brake retardation was similar. Therefore, increasing the number of cars that brake before the collision could avoid accidents or at least reduce the accident symptoms. In $20 \%$ of the cases the driver did not show a measurable reaction before the collision. An earlier recognition of possibly dangerous situations enlarges the window of opportunity to intervene e.g. by braking which could avoid accidents or at least reduce their severity.

\section{B. Detailed Accident Reports}

The second data source consists of 4.500 reports of local accidents with pedestrians collected by the police of Braunschweig. These reports include information of the accidents which are analysed with regard to the behavior of the car drivers and pedestrians which caused the accident. [4] At first we filtered out all accidents without pedestrians, which left 63 reports that could be used in the following analysis. All these accidents took place whithin urban areas.

To evaluate the reasons for accidents with pedestrians, we considered the age of the pedestrians, the location and the cause of the accident regarding who was at fault. The results implies, that in 22 of those cases, the driver was at fault. By contrast, the pedestrians had caused the accident in 41 of all cases. In those cases where the pedestrians had caused the accident, they were most often either below 18 years of age or 60 years and older (see fig. 3).

Often pedestrians did not check for traffic or ignored the traffic lights. This generally happend at straight roads. If the drivers were at fault, the accident happend mostly at intersections or crossways.

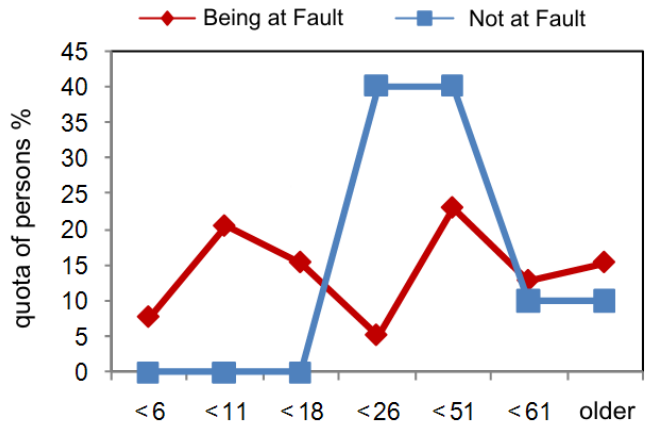

Figure 3. Fault-Age-Distribution of accidents happend in urban areas

\section{Scenarios}

In the previous analysis we summarized the circumstances of typical accidents. In this section, we will build general scenarios within the previously collected knowledge. They will be used later to determine on effective means to prevent such accidents in the future.

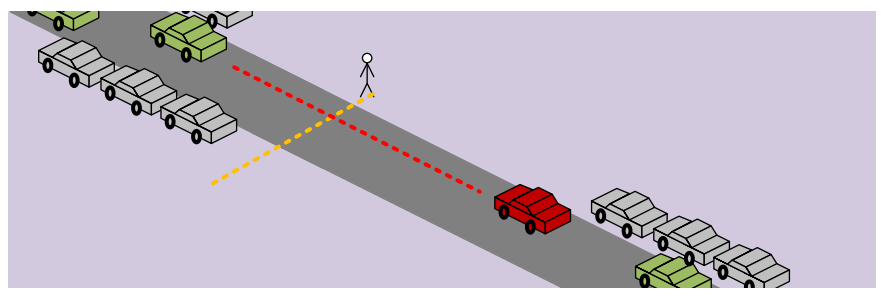

Figure 4. Scenario "straight road"

One of the simplest but most occurring type of accident is shown in Figure 4. Here a car is driving along a straight road in a urban area. Suddenly, a pedestrian steps off the sidewalk on the road without taking care of the approaching car. The car has to brake very fast or perform an evasive maneuver if possible. Parking cars on the sides of the roads increase the risk in this scenario.

Figure 5 shows a crossway with traffic lights in a urban area. The cars have the right of way at the moment and the pedestrian has to wait. Suddenly, the pedestrian decides to ignore the traffic lights and starts crossing the road. The car has to brake very fast or do a change maneuver if possible.

Scenario "crossway" (Figure 6) is based on the scenario "crossway turn right", but in this case the car has to consider the pedestrian who wants to cross the road. If the driver does not recognize the pedestrian, an accident will happen.

Until now we have described typical scenarios with one pedestrian only. In the following, we would like to add two scenarios which are more difficult to handle. One of those scenarios is "crowded area", shown in Figure 7. It is located at a place where many people are crossing the road frequently. There are several situations which resemble the "crowded area" scenario:

- After school: Many children come out of the school at the same time. They are running and/or playing without taking notice of the traffic. 


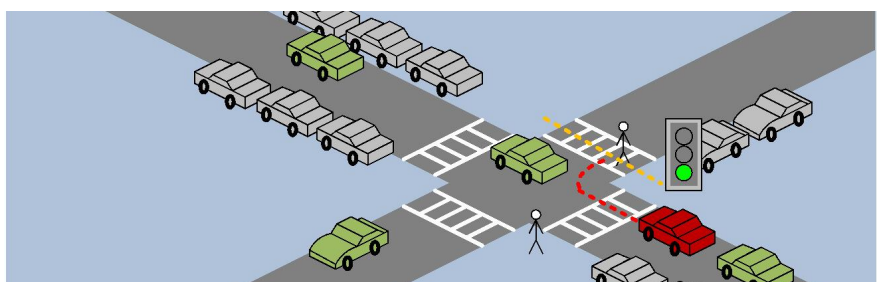

Figure 5. Scenario "crossway turn right"

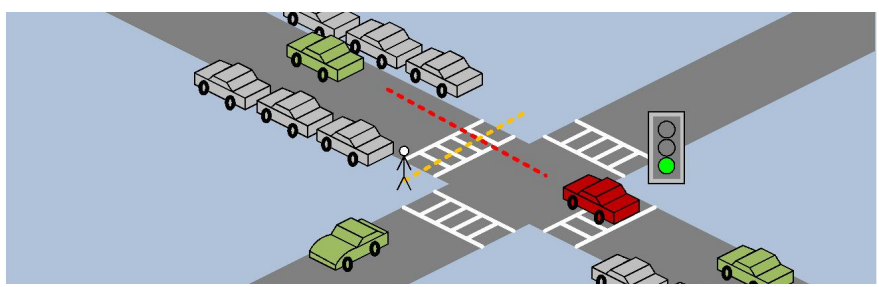

Figure 6. Scenario "crossway"

- Around a home for the aged: Old people are leaving the building at random and crossing a road without taking notice of the traffic.

- A person tries to catch a bus at a busstop, but is late and crosses the street without taking notice of the traffic. Most times deboarding people are careless too.

- At a mall or a shopping center: Customers are crossing the street without taking notice of the traffic.

In all of these cases, the people do not take care of the traffic and may cross a road in a hurry. This is one of the most unpredictable scenarios and difficult to estimate for the driver.

Scenario "parking lot" (Figure 8) is a special case of the "crowded area". In this case the driver wants to maneuver his car and has to keep the different directions in his view. Many pedestrians walk around the car and may be in the blind angle. They could suddenly appear in front or back of the car. The car should not move or has to brake if a pedestrian is in the direction of motion. Children are more at risk than adults in this situation, because of their body height.

The next two scenarios does not appear very often in the accident statistics, but for the pedestrians these types of accidents are in most cases deadly. Therefore, they will be considered for the completeness of this analysis.

Figure 9 shows the scenario "highway" with a person on a curb of a highway. His car has broken down and now he waits for a service car. The other cars are driving very fast with speeds exceeding $100 \mathrm{~km} / \mathrm{h}$ and do not expect a pedestrian on the road.

The scenario "country road" is shown in Figure 10. Here a pedestrian is walking along a bendy country road where he is not very visible and hard to recognize. This could be even worse under conditions such as fog, heavy rain or at night. The cars are driving with approximately $100 \mathrm{~km} / \mathrm{h}$ passing the pedestrian. Additionally, instead of walking along the road, the pedestrian would be a wanderer and exit a trail which crosses the road. The pedestrian could be hidden by plants being nearly invisible for the driver until he steps on the road.



Figure 7. Scenario "crowded area"



Figure 8. Scenario "parking lot"

\section{Summary}

Most accidents happen in urban areas on straight roads without intersections or crossways. This is backed by results from the GIDAS study. Most times the pedestrian suddenly stepped on the lane without a previous indication and did not recognize the car. In some of these cases, the pedestrians were partly hidden by parking cars. It has to be mentioned that the drivers did not brake at all in 50\% of those cases. In nearly all cases the brake retardation did not reached the physical minimum. The local accident study figured out, that in $2 / 3$ of the cases the pedestrians caused the accident. Most times the pedestrians suddenly stepped on the lane without paying attention to the traffic. This is typically a problem on straight roads where the drivers did not expect a pedestrian. In addition, the study showed a peak of accidents with old people and children. This scenario matches to approximately $29 \%$ of all accidents between cars and pedestrians (Figure 4).

The second significant type of accidents happens at crossways or intersections when a car wants to turn. A traffic light shows green for pedestrians crossing the trajectory of the car. The driver has to wait in this situation but overlooks the pedestrian. According to the GIDAS study, $23.6 \%$ of all accidents happen at crossways and also the local study shows a peak of accidents while cars want to turn. In contrast to straight roads, where the pedestrians caused the accidents, here the driver did not recognize pedestrians. Old poeple and children are not so much involved in these cases as has been the case at straight roads.

Both previously described scenarios are situated in urban areas, where pedestrians and cars converge very often. They have been characterized by their most significant attributes and will be used in section $\mathrm{V}$ as a base for further efforts on pedestrian safety.

\section{RELATED WORK}

There are two types of measures for the protection of pedestrians. Those which reduce the consequences of accidents and preventive measures. Nowadays the reduction of 


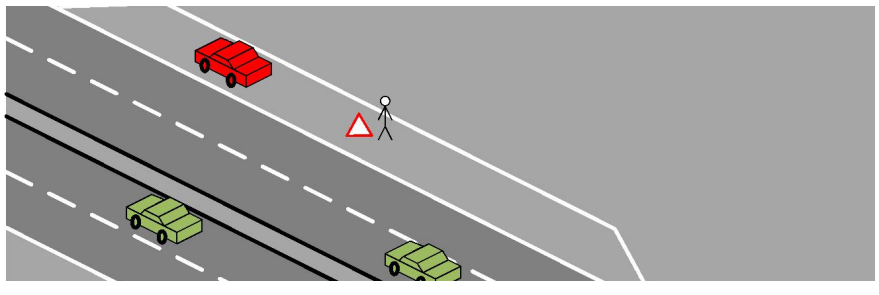

Figure 9. Scenario "highway"

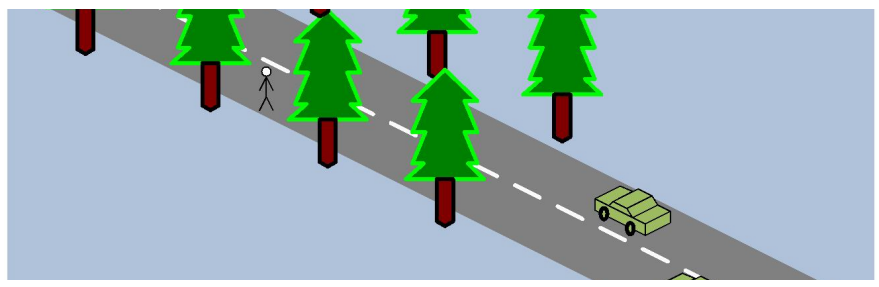

Figure 10. Scenario "country road"

implications is already implemented by a special design of the autobody [5]. In contrast to that, current research in the field of preventive measures focus on sensors built into the car to detect obstacles or improve the view of the driver e.g. with a nightvision camera. The EU-project PROTECTOR [6] and the project Aktiv-AS [7] of the Federal Ministry of Economics in Germany use laser scanner, radar and camera systems to detect pedestrians or cyclists and try to identify a critical situation with complex algorithms. In those cases the system can take actions like warn the driver or amplify the break booster to shorten the stopping distance. Also there is a project called AMULETT [8] which aims at identifying critical situations by using a transponder carried by pedestrians as a location device.

All mentioned projects avoid a possible collision just by asserting the position of pedestrians through complex sensor devices. There is no bidirectional exchange of information between the pedestrian and the car. But extended information like the trajectory or the change of speed could lead to a reliable prediction of collisions. In the next section, a system architecture will be introduced which uses communication between the road users to predict and prevent accidents.

\section{CONCEPT}

In section II we discussed the general accident situation and common mistakes done by pedestrians and drivers. To determine effective means to prevent such accidents in the future, we have to figure out how to improve measures for safety of pedestrians. Reduction of the implications of accidents are well-researched. Therefore, this paper will focus on preventive measures.

To prevent an accident we have four options: raise the attention of the road user, focus the attention, announce a upcoming collision or take over the control of the car (emergency break / evasive maneuver). The simplest way to raise the attention is to warn a road user every time he enters an area which is marked as very risky. This is easy to realize by a software upgrade in common GPS systems, but on the other hand this only offers little protection. Furthermore, it is possible that the users tend to switch off the device or ignore it since too many warnings are generated.

What we need for all preventive measures is a reliable indicator for critical situations which would trigger a counteractive measure. Such an indicator could be realized by tracking the road users and predict their trajectory. Every time two trajectories impend to intersect, an alert must be given.

The idea presented in this paper is to use existing technology, e.g. GPS, Wireless LAN or UWB built in a mobile device to integrate the road users into a cooperative traffic system. Such a system should detect critical situations and induce counteractive measures such as warn the road user. Each device carried by the pedestrian or integrated into the car should meet at least the two requirements localization and communication.

\section{A. Localization}

To track each road user, he must be visible to the cooperative traffic system. This requires at least the global or the relative position of the road users reported to a centralized system or all neighboring road users. If a road user has been tracked, his trajectory could be predicted and a evaluation of the situation is possible. If the trajectory impend to intersect with another object a counteractive measure could be performed.

\section{B. Communication}

A cooperative traffic system is an inherently distributed system. Each road user in such a system would be a part of it. To reach a global view of all objects it is necessary to transfer information about the road users (e.g. the position, moving direction and speed of the road user) to a central system or the neighboring devices. In addition, communication could be used to trigger alerts to the road users, if someone else detected a critical situation.

\section{Proposed System Architecture}

Based on the previous discussion and results, we designed three different system architectures with varying level of capabilities and complexity. This section introduces these system architectures, which may assist road users in critical situations. Especially if the line of sight between road users is blocked by static or moveable obstacles, this system assist the road user. Every time a critical situation is detected, an alert to the involved road users will be given. In addition, other safety activities could be initiated, e.g. prepare the break booster to shorten the stopping distance.

\section{A. System 1}

The goal of the first presented system architecture is a simple detection of the own position, which is subsequently distributed to the neighboring user equipment devices (UED). With the own information and the collected data of the environment, a detection of an upcoming collision can be realized. In such a case the UED should initiate an alert, which could raise or focus the attention of the road user. 


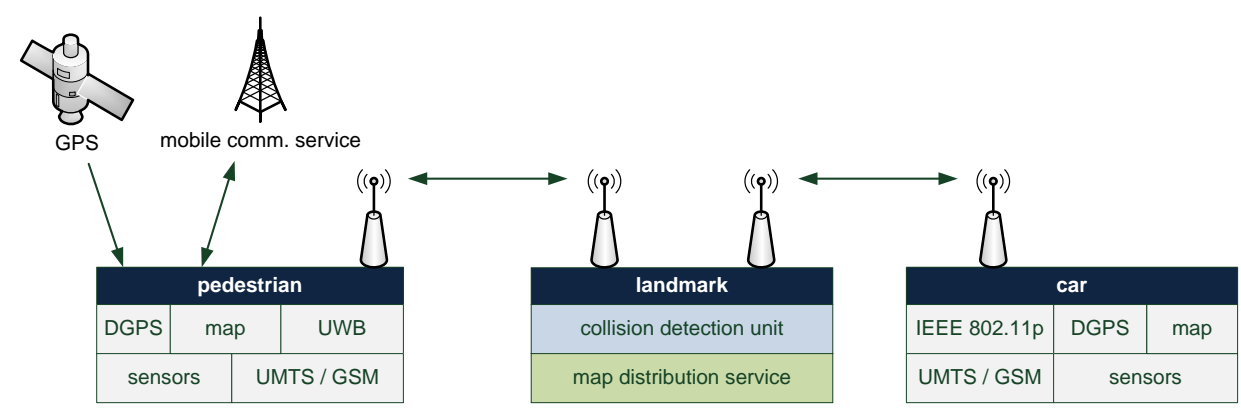

Figure 11. Proposed system for urban areas.

A UED acquires its own position by a common GPS receiver which is combined with GSM triangulation and a map matching technology [9] to gain a more precise localization. A low cost inertial measurement unit (IMU) [10] in the UED is used to detect spontaneous changes of directions. The expected accuracy is around 5-10 meters. The gained position will be transmitted to neighboring UEDs using an IEEE 802.11p interface in ad-hoc mode. In addition, the moving direction or routing information could be transmitted to improve the prediction of collisions. The standard IEEE $802.11 \mathrm{p}$ provides a high coverage range up to $300 \mathrm{~m}$ and also works at higher moving speeds up to $160 \mathrm{~km} / \mathrm{h}$. An average latency below $50 \mathrm{~ms}$ [11] is an advantage over common mobile communication systems like GSM or UMTS [12]. Furthermore, it provides a more than sufficient bandwidth of $54 \mathrm{Mbit} / \mathrm{s}$ and reserved channels for emergency communication.

We expect to mitigate at least the scenarios "highway" and "country road" with this system, but the benefit in urban scenarios is questionable. To detect a collision each UED in the neighborhood must be tracked and each UED has to match his own predicted trajectory with all received trajectories. Thus, a high demand for processing power is expected, if the number of UED raises. Also the localization might not precise enough to differ between a curbstone and the road.

\section{B. System 2}

To improve the localization of system 1, the GPS receiver is extended by DGPS which provides an accuracy below one meter. The relative position can be determined by a Ultra Wideband (UWB) signal [13] and an antenna array [14], which can be installed at the front of vehicles. The communication between the UEDs is realized through IEEE $802.11 \mathrm{p}$ or UWB which provides a range of approximately $10 \mathrm{~m}$ [15]. The integrated GSM module may be used for long distance transmissions.

In addition to the scenarios "highway" and "country road", the refined localization can mitigate the scenarios "straight road", "crossway turn right" and "crossway". Referring to section II, this system fits to over 55\% of all accidents. However, this system still lacks scalability and will run into trouble at crowded areas were many pedestrians are to be tracked.

\section{System 3}

The main disadvantages of the previous systems are low accuracy and the lack of scalability. With the third system approach, we would like to introduce a more complex architecture which is able to fulfill both requirements for all scenarios.

Figure 11 shows the architecture of the proposed system. There are two UEDs, one carried by a pedestrian (left) and one installed in a car (right). Both are communicating with a landmark, which could be installed at critical areas (e.g. crossways, intersections or parking lots of malls). A landmark holds miscellaneous types of communication interfaces to support different ways to communicate to several UEDs. Thus, it is possible to use short-range and low-powered communication technology for pedestrians and long-distance technology to communicate with cars. The build-in collision detection unit detects collisions or critical situations based on the data received from UEDs. With additional information like the predicted trajectory, speed or context information, a more precise prediction is possible. If a critical situation is detected, an alert can be transmitted to the neighboring UEDs including information about involved parties.

Each UED has at least a component to locate itself and a component for communication with the landmark or with other UEDs. Depending on the class of the device (pedestrian, car or cyclist) it can hold several sensors to locate itself, identify a context or predict the trajectory. UWB is a potential technology for a UED carried by a pedestrian. In addition to good characteristics for communication, UWB is able to locate other devices. To gain a very precise localization with low delay it could be combined with a low cost IMU and a map matching technology, which uses a high resolution map received from the map distribution service of the landmark. In addition, a DGPS module can refine the own position. [16]

We expect that IEEE $802.11 \mathrm{p}$ will be a part of future vehicle-2-vehicle systems to exchange and forward messages. Hence, we choosed this standard for the communication between cars and the landmark. Like in the UEDs for pedestrians, a UED in the car also uses different sensors, a map matching system and DGPS to predict the own trajectory.

\section{CONCLUSION}

In this paper a accident study based on the GIDAS database and local police reports has been done. Both sources led to the 
conclusion, that most of the accidents occur at straight roads in urban areas. Crossways could be identified as the second significant scenario for many accidents with pedestrians. In both cases the pedestrians or the driver did not recognize the other road user or misjudged the situation.

To provide an effective means to prevent such accidents in the future, three system architectures were proposed in this paper. All of them should reduce the number of accidents by raising the attention of the road users in case of an upcoming critical situation. But they differ in complexity and area of application. While System 1 is good at rural areas and does not need additional infrastructure, it does not scale well at crowded areas and is not able to decide if a person stands on a road or on the sidewalk. The low accuracy is improved in System 2, which is still independent of additional infrastructure. Thus, this architecture is good enough to provide pedestrian safety in urban areas without dense traffic. In crowded areas there are many people to track. Such clouds of UEDs are very hard to track in a completely distributed way. A single UED could be overstrained to track his neighborhood and execute collision algorithms on them. To mitigate this situation, a landmark is introduced in system 3 . Such a landmark could be installed at crowded areas and should assist the UEDs which do not need to be very powerful. In addition, the communication channel is less stressed if the landmark provides most of the required information for all UEDs over multicast.

Finally, extended information transmitted by a UED could lead to a reliable and accurate prediction of collisions. The prevention of the most occurring accidents or at least mitigate the accident symptoms with such technology is possible.

\section{REFERENCES}

[1] Bundesministerium für Verkehr, Bau-, und Wohnungswesen, Ed., Verkehr in Zahlen 2003/2004. Deutscher Verkehrs-Verlag, 2003.

[2] "GIDAS - German In-Depth Accident Study," 2008. [Online]. Available: http://www.gidas.org/

[3] M. Vollrath, S. Briest, and J. Drewes, Ableitung von Anforderungen an Fahrerassistenzsysteme aus Sicht der Verkehrssicherheit. Berichte der Bundesanstalt für Straßenwesen, Fahrzeugtechnik, Heft F 60. Wirtschaftsverlag NW, Bremerhaven, 2006.

[4] S. Briest and M. Vollrath, "In welchen Situationen machen Fahrer welche Fehler? Ableitung von Anforderungen an Fahrerassistenzsysteme durch In-Depth-Unfallanalysen," in Integrierte Sicherheit und Fahrerassistenzsysteme, VDI, Ed. Wolfsburg: VDI, 2006, pp. 449-463.

[5] Aachen, Forschungsgesellschaft Kraftfahrwesen mbH, "Pedestrian Protection," 2005. [Online]. Available: http://www.ika.rwth-aachen.de/ pdf_eb/gb2-08e_pedestrian_protection.pdf

[6] _ "Protector," 2005. [Online]. Available: http://www.ika. rwth-aachen.de/pdf_eb/gb6-10e_protector.pdf

[7] "Adaptive und kooperative Technologien für den intelligenten Verkehr," 2009. [Online]. Available: http://www.aktiv-online.org/

[8] Pedestrian Protection Systems using Cooperative Sensor Technology. 11th Internatinal Forum on Advanced Microsystems for Automotive Applications AMAA, 2007.

[9] I. Spassov, M. Bierlaire, and B. Merminod, "Map-based Autonomous Personal Localization and Tracking," TRISTAN VI, 2007.

[10] S. Godha and M. E. Cannon, "Integration of DGPS with a Low Cost MEMS - Based Inertial Measurement Unit (IMU) for Land Vehicle Navigation Application," ION GPS -05, September13-16, 2005, Long Beach, CA.

[11] S. Eichler, "Performance evaluation of the IEEE 802.11p WAVE communication standard," 2007, pp. 2199-2203, cited By (since 1996): 1. [Online]. Available: www.scopus.com

[12] C. Ribeiro, "Bringing Wireless Access to the Automobile: A Comparison of Wi-Fi, WiMAX, MBWA, and 3G," 2005. [Online]. Available: http://www.rh.edu/ rhb/cs_seminar_2005/SessionB3/ribeiro.pdf
[13] M. Tüchler, "Location Accuracy of an UWB localization system in a mulit-path enviroment," IEEE Conference on Ultra-Wideband, 2005.

[14] J. Shi, H. Mueller, and M.Marx, "Pedestrian Detection and Localization Using Antenna Array and Sequential Triangulation," IEEE Conference on Intelligent Transportation Systems, 2005.

[15] "ECMA-368: High Rate Ultra Wideband PHY and MAC Standard," Geneva, Switzerland: ECMA, 2008. [Online]. Available: www. ecma-international.org/publications/files/ECMA-ST/ECMA-368.pdf

[16] M. Kourogi et al., "Indoor/Outdoor Pedestrian Navigation with an Embedded GPS/RFID/Selfcontained Sensor System," ICAT, 2006. 